\title{
Variation in haemodynamic monitoring for major surgery in European nations: secondary analysis of the EuSOS dataset
}

Tahania Ahmad $^{1 \dagger}$, Christian M. Beilstein ${ }^{1 \dagger}$, Cesar Aldecoa ${ }^{2}$, Rui P. Moreno ${ }^{3}$, Zsolt Molnár ${ }^{4}$, Vesna Novak-Jankovic ${ }^{5}$, Christoph K. Hofer ${ }^{6}$, Michael Sander ${ }^{7}$, Andrew Rhodes $^{8}$ and Rupert M. Pearse ${ }^{1,9^{*}}$

\begin{abstract}
Background: The use of cardiac output monitoring may improve patient outcomes after major surgery. However, little is known about the use of this technology across nations.

Methods: This is a secondary analysis of a previously published observational study. Patients aged 16 years and over undergoing major non-cardiac surgery in a 7-day period in April 2011 were included into this analysis. The objective is to describe prevalence and type of cardiac output monitoring used in major surgery in Europe.

Results: Included in the analysis were 12,170 patients from the surgical services of 426 hospitals in 28 European nations. One thousand four hundred and sixteen patients (11.6\%) were exposed to cardiac output monitoring, and 2343 patients (19.3\%) received a central venous catheter. Patients with higher American Society of Anesthesiologists (ASA) scores were more frequently exposed to cardiac output monitoring (ASA I and II, 643 patients [8.6\%]; ASA III-V, 768 patients [16.2\%]; $p<0.01$ ) and central venous catheter (ASA I and II, 874 patients [11.8\%]; ASA III-V, 1463 patients [30.9\%]; $p<0.01$ ). In elective surgery, 990 patients (10.8\%) were exposed to cardiac output monitoring, in urgent surgery 252 patients (11.7\%) and in emergency surgery 173 patients (19.8\%). A central venous catheter was used in 1514 patients (16.6\%) undergoing elective, in 480 patients (22.2\%) undergoing urgent and in 349 patients (39.9\%) undergoing emergency surgery. Nine hundred sixty patients (7.9\%) were monitored using arterial waveform analysis, 238 patients (2.0 \%) using oesophageal Doppler ultrasound, 55 patients $(0.5 \%)$ using a pulmonary artery catheter and 44 patients (2.0 \%) using other technologies. Across nations, cardiac output monitoring use varied from $0.0 \%$ (0/249 patients) to $27.5 \%$ (19/69 patients), whilst central venous catheter use varied from $5.6 \%$ (7/125 patients) to $43.2 \%$ (16/37 patients).
\end{abstract}

Conclusions: One in ten patients undergoing major surgery is exposed to cardiac output monitoring whilst one in five receives a central venous catheter. The use of both technologies varies widely across Europe.

Trial registration: ClinicalTrials.gov Identifier: NCT01203605. Date of registration: 15.09.2010.

Keywords: Major surgery, Perioperative medicine, Cardiac output monitoring, Haemodynamic monitoring

\footnotetext{
*Correspondence: r.pearse@qmul.ac.uk

${ }^{\dagger}$ Equal contributors

'Barts and The London School of Medicine and Dentistry, Queen Mary University of London, London, UK

${ }^{9}$ Adult Critical Care Unit, Royal London Hospital, London E1 1BB, UK

Full list of author information is available at the end of the article
} 


\section{Background}

More than 312 million surgical procedures are performed worldwide each year with an estimated mortality between 1 and $4 \%$ [1, 2]. Postoperative complications and death are most frequent amongst high-risk patients, who are older, have co-morbid disease and undergo major surgery [3]. The dose of intravenous fluid and vasoactive drugs has an important effect on patient outcomes following major gastrointestinal surgery [4]. These treatments are prescribed according to subjective criteria leading to wide variation in clinical practice [5-9]. One potential solution to this problem is the use of cardiac output monitoring to guide administration of intravenous fluid and vasoactive drugs [4].

The use of perioperative cardiac output monitoring remains controversial, with differing interpretations of the evidence base for this treatment approach [10-18]. Recent evidence from a large clinical trial suggests that the benefit associated with this technology may be more marginal than previously believed, whilst some commentators have raised safety concerns $[4,19,20]$. Cardiac output monitoring has been recommended for patients undergoing selected types of major surgery both by the National Institute for Health and Care Excellence (NICE) in the UK and in a report commissioned by the Centers for Medicare and Medicaid Services in the USA [21, 22]. However, clinician surveys and anecdotal evidence suggest there is wide variation in the use of this technology [6-9].

Across Europe, little is known about the use of cardiac output monitoring in non-cardiac surgery. A previously published 7-day cohort study described mortality and perioperative care in 28 European nations (European Surgical Outcomes Study [EuSOS]) [2]. Data were collected which describe the use of cardiac output monitoring and central venous catheterisation. We performed a secondary analysis of the EuSOS dataset to describe the prevalence and types of cardiac output monitoring in patients undergoing major surgery across European nations.

\section{Methods}

\section{Ethics}

Ethics requirements differed by country. In Denmark, centres were exempt from ethics approval as the study was deemed to be a clinical audit. In all other nations, formal ethics approval was obtained. For the United Kingdom, approval was given by the Oxfordshire Research Ethics Committee B (Harrow, United Kingdom, 15th November 2010, chair Prof. M. Rees, reference number 10/H0605/72). In Finland, informed consent was obtained for all participants as required by the ethics committee.

\section{Setting}

This is a secondary analysis of the previously published European Surgical Outcomes Study, a 7-day observational cohort study including consecutive patients undergoing inpatient non-cardiac surgery in April 2011 [2].

\section{Data collection}

Eligible for EuSOS were consenting patients aged 16 years and over undergoing inpatient non-cardiac surgery, irrespective of the chosen anaesthetic technique. We excluded patients undergoing planned day-case surgery, cardiac surgery, neurosurgery, radiological or obstetric procedures. From this dataset, we only included patients undergoing major surgery (duration more than $90 \mathrm{~min}$ as defined in the EuSOS protocol) in this analysis. Use of haemodynamic monitoring in the operating room was recorded on a paper case record form in the following categories: central venous catheter, arterial waveform analysis (including both calibrated and uncalibrated analysis), oesophageal Doppler ultrasound, pulmonary artery catheter and other. Data were assessed for completeness and checked for plausibility and consistency. A list of participating hospitals and full details of the methodology of the study can be found in the original publication [2].

\section{Statistical analysis}

A descriptive analysis was carried out according to a prospectively written statistical analysis plan. Data are presented as mean (standard deviation) for continuous variables and absolute or relative frequencies as percentages for categorical variables. Baseline characteristics of patient groups were tabulated and differences between groups compared using Pearson's chi-square $\left(\chi^{2}\right)$ test or $t$-test as appropriate. Prevalence of each type of haemodynamic monitoring was assessed per urgency of surgery, surgical speciality and per nation. Countries with less than ten recruited patients were excluded from graphs presenting per nation data. All analysis was carried out using STATA MP 13.1 (STATA Corp, USA).

\section{Results}

\section{Baseline characteristics}

From the original EuSOS dataset (46,539 patients), we excluded 34,349 patients who did not undergo major surgery and 20 patients for missing haemodynamic monitoring data. In total, 12,170 patients who underwent major surgery in 426 hospitals from 28 countries were included into this analysis. The patient flow diagram is available as an additional online file (Additional file 1: Figure S1). Baseline characteristics of patients are presented in Table 1 according to exposure to cardiac output monitoring and central venous catheter (CVC). 
Table 1 Baseline characteristics of patients without/with cardiac output monitoring and with central venous catheter

\begin{tabular}{|c|c|c|c|c|c|}
\hline & All & $\begin{array}{l}\text { Without cardiac output } \\
\text { monitoring }\end{array}$ & $\begin{array}{l}\text { With cardiac output } \\
\text { monitoring }\end{array}$ & $p^{*}$ & With central venous catheter \\
\hline Age & & & & $<0.01$ & \\
\hline Mean (SD) & $61.7(16.5)$ & $61.4(16.6)$ & $63.6(15.2)$ & & $62.9(15.0)$ \\
\hline Gender & & & & $<0.01$ & \\
\hline Male & $6290(51.7 \%)$ & $5706(90.7 \%)$ & $584(9.3 \%)$ & & $935(14.9 \%)$ \\
\hline Female & $5878(48.3 \%)$ & $5048(85.9 \%)$ & $830(14.1 \%)$ & & 1407 (23.9\%) \\
\hline Current smoker & 2329 (19.3\%) & 2041 (19.1\%) & $288(20.5 \%)$ & 0.20 & $527(22.7 \%)$ \\
\hline ASA Score & & & & $<0.01$ & \\
\hline | and || & $7400(60.8 \%)$ & $6793(91.4 \%)$ & $643(8.6 \%)$ & & $874(11.8 \%)$ \\
\hline III, IV and V & 4736 (39.0 \%) & 3978 (84.0 \%) & $768(16.2 \%)$ & & 1463 (30.9 \%) \\
\hline Urgency of surgery & & & & $<0.01$ & \\
\hline Elective & $9132(75.1 \%)$ & $8142(89.2 \%)$ & $990(10.8 \%)$ & & $1514(16.6 \%)$ \\
\hline Urgent & $2161(17.8 \%)$ & 1909 (88.3 \%) & $252(11.7 \%)$ & & $480(22.2 \%)$ \\
\hline Emergency & $875(7.2 \%)$ & $702(80.2 \%)$ & $173(19.8 \%)$ & & 349 (39.9 \%) \\
\hline Surgical speciality & & & & $<0.01$ & \\
\hline Orthopaedic & 3984 (32.8 \%) & $3800(95.4 \%)$ & $184(4.6 \%)$ & & $175(4.4 \%)$ \\
\hline Breast & $350(2.9 \%)$ & 331 (94.6 \%) & $19(5.4 \%)$ & & $32(9.2 \%)$ \\
\hline Gynaecology & $1051(8.7 \%)$ & 1005 (95.6 \%) & $46(4.4 \%)$ & & $70(6.7 \%)$ \\
\hline Vascular & $807(6.7 \%)$ & $633(78.4 \%)$ & $174(21.6 \%)$ & & $267(33.3 \%)$ \\
\hline Gastrointestinal & 2717 (22.3 \%) & 2144 (78.9 \%) & $573(21.1 \%)$ & & 971 (35.7\%) \\
\hline Hepato-biliary & $510(4.2 \%)$ & $370(72.6 \%)$ & $140(27.5 \%)$ & & $299(58.6 \%)$ \\
\hline Plastic or cutaneous & $192(1.6 \%)$ & $188(97.9 \%)$ & $4(2.1 \%)$ & & $22(11.5 \%)$ \\
\hline Urology and kidney & 1219 (10.0 \%) & 1073 (87.1\%) & $146(12.9 \%)$ & & $266(28.2 \%)$ \\
\hline Head and neck & $684(5.6 \%)$ & $638(93.3 \%)$ & $46(6.7 \%)$ & & $64(9.4 \%)$ \\
\hline Other & $622(5.1)$ & $542(87.1 \%)$ & 80 (12.9\%) & & $167(26.9 \%)$ \\
\hline \multicolumn{6}{|l|}{ Co-morbid disorder } \\
\hline No co-morbid disorder & 6679 (54.9\%) & 6095 (91.2 \%) & $584(8.7 \%)$ & $<0.01$ & $918(13.7 \%)$ \\
\hline Cirrhosis & 186 (1.5\%) & $126(67.7 \%)$ & $60(32.3 \%)$ & $<0.01$ & 97 (52.2 \%) \\
\hline Congestive heart failure & 646 (5.3 \%) & $519(80.3 \%)$ & $127(19.7 \%)$ & $<0.01$ & $196(30.3 \%)$ \\
\hline COPD & 1590 (13.1\%) & 1352 (85.0 \%) & $238(15.0 \%)$ & $<0.01$ & $388(24.4 \%)$ \\
\hline Coronary artery disease & 1999 (16.5\%) & $1685(84.3 \%)$ & $314(15.7 \%)$ & $<0.01$ & $460(23.1 \%)$ \\
\hline NIDDM & 1119 (9.2 \%) & $973(87.0 \%)$ & $146(13.1 \%)$ & 0.12 & $245(21.9 \%)$ \\
\hline Metastatic cancer & 1014 (8.4 \%) & 815 (80.4 \%) & 199 (19.6\%) & $<0.01$ & $421(41.5 \%)$ \\
\hline Stroke & 719 (5.9\%) & $601(83.6 \%)$ & $118(16.4 \%)$ & $<0.01$ & $172(23.9 \%)$ \\
\hline \multicolumn{6}{|l|}{ Hospital statistics } \\
\hline Length of hospital stay (mean, SD) & $8.9(9.4)$ & $8.5(9.3)$ & $12.4(12.0)$ & $<0.01$ & $15.0(12.9)$ \\
\hline ICU admission & 2534 (20.8 \%) & 1900 (17.7 \%) & $634(44.8 \%)$ & $<0.01$ & $1445(61.7 \%)$ \\
\hline Mortality & $667(5.5 \%)$ & $535(5.0 \%)$ & $142(10.0 \%)$ & $<0.01$ & $287(12.2 \%)$ \\
\hline Total & 12170 & $10,754(88.4 \%)$ & $1416(11.6 \%)$ & & $2343(19.3 \%)$ \\
\hline
\end{tabular}

Data presented as mean (SD) or $n$ (\%)

ASA American Society of Anesthesiologists, ICU intensive care unit, NIDDM non-insulin-dependent diabetes mellitus, COPD chronic obstructive pulmonary disease ${ }^{*} p$ value describes comparison of the patient groups with and without cardiac output monitoring

Use of haemodynamic monitoring

One thousand four hundred and sixteen patients (11.6\%) were exposed to cardiac output monitoring, and
2343 (19.3 \%) patients received a CVC. Six hundred eighty $(5.6 \%)$ patients received both cardiac output monitoring and a CVC. Patients exposed to cardiac 
output monitoring or central venous catheterisation had higher American Society of Anesthesiologists (ASA) scores, were slightly older and more likely to undergo urgent or emergency surgery (Table 1). Patients receiving cardiac output monitoring or a CVC had a longer hospital stay, were more likely to be admitted to intensive care and were more likely to die than patients who did not receive cardiac output monitoring (Table 1). Nine hundred sixty patients $(7.9 \%)$ were monitored using arterial waveform analysis, 238 (2.0 \%) using oesophageal Doppler ultrasound, 55 patients (0.5 \%) using a pulmonary artery catheter and 44 patients (2.0\%) using another technology. Arterial waveform analysis was the most frequently used technique across urgencies of surgery (Fig. 1), surgical specialities (Fig. 2) and nations. Country-level data describing the use of different monitoring techniques is available as an additional online file (see Additional file 1: Figure S2 and Table S1). Across European nations, cardiac output monitoring use varied from $0.0 \%$ (0/249 patients) to $27.5 \%$ (19/69 patients) and the use of CVC from $5.6 \%$ (7/125 patients) to $43.2 \%$ (16/37 patients) (Fig. 3).

\section{Discussion}

The principal finding of this analysis was that one in ten patients undergoing major surgery is exposed to cardiac output monitoring and one in five patients receives a CVC. Cardiac output monitoring and CVC use were more frequent with increasing ASA score and increasing urgency of surgery. The use of both cardiac output monitoring and CVCs was associated with a longer hospital stay, more admissions to critical care and higher mortality. Importantly, there was wide variation in the use of cardiac output monitoring and CVCs across European nations. The most commonly used method of cardiac output monitoring was arterial waveform analysis.

There is very little published patient-level data describing the use of cardiac output monitoring either in individual countries or at an international level. However, questionnaire-based surveys amongst anaesthesiologists have been used to explore the use of cardiac output monitoring in high-risk surgical patients. The most widely cited of these is an online questionnaire survey conducted in 2011 by Cannesson et al. amongst members of the American Society of Anesthesiologists and the European Society of Anaesthesiology. From 368 respondents, $35 \%$ routinely monitored cardiac output with similar numbers in Europe and in North America [6]. A similar survey of members of the Korean Society of Anesthesiologists revealed that $59 \%$ of 139 respondents were using cardiac output monitoring [8]. The findings of a paper-based survey of Chinese anaesthetists showed that cardiac output monitoring was used by $13 \%$ of 210 respondents [9]. In addition to the variation in use of cardiac output monitoring, these surveys also reveal differences in the monitoring technique used. This variability is supported by another online questionnaire-based survey, exploring the use of goal-directed therapy in major elective surgery amongst members of the Association of Anaesthetists of Great Britain and Ireland, the American Society of Anesthesiologists and the Australia and New Zealand College of Anaesthetists [7]. Overall, the findings of these clinician surveys are consistent with the findings of our analysis of patient-level data. The lower use of cardiac output monitoring in our data compared to the data provided by Cannesson et al. might be due to the broader inclusion criteria of our study [6]. Suggested explanations for the wide variation in clinical practice were local factors

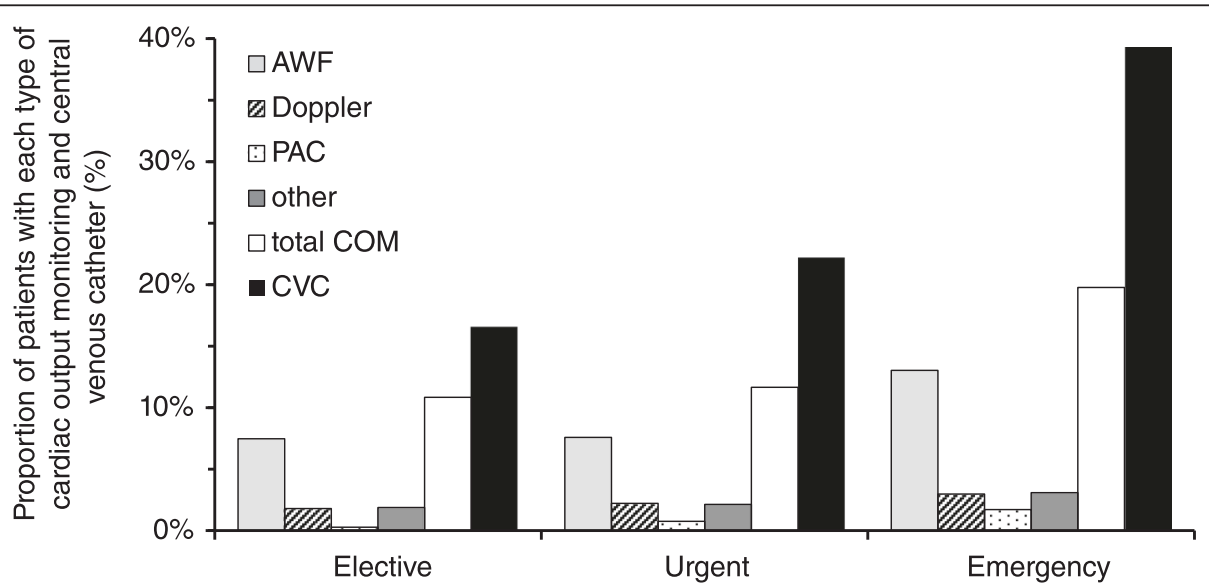

Fig. 1 Use of cardiac output monitoring and central venous catheter per urgency of surgery. Data displayed as percentage per urgency of surgery. AWF arterial waveform analysis, Doppler Doppler ultrasound, PAC pulmonary artery catheter, COM cardiac output monitoring, CVC central venous catheter 


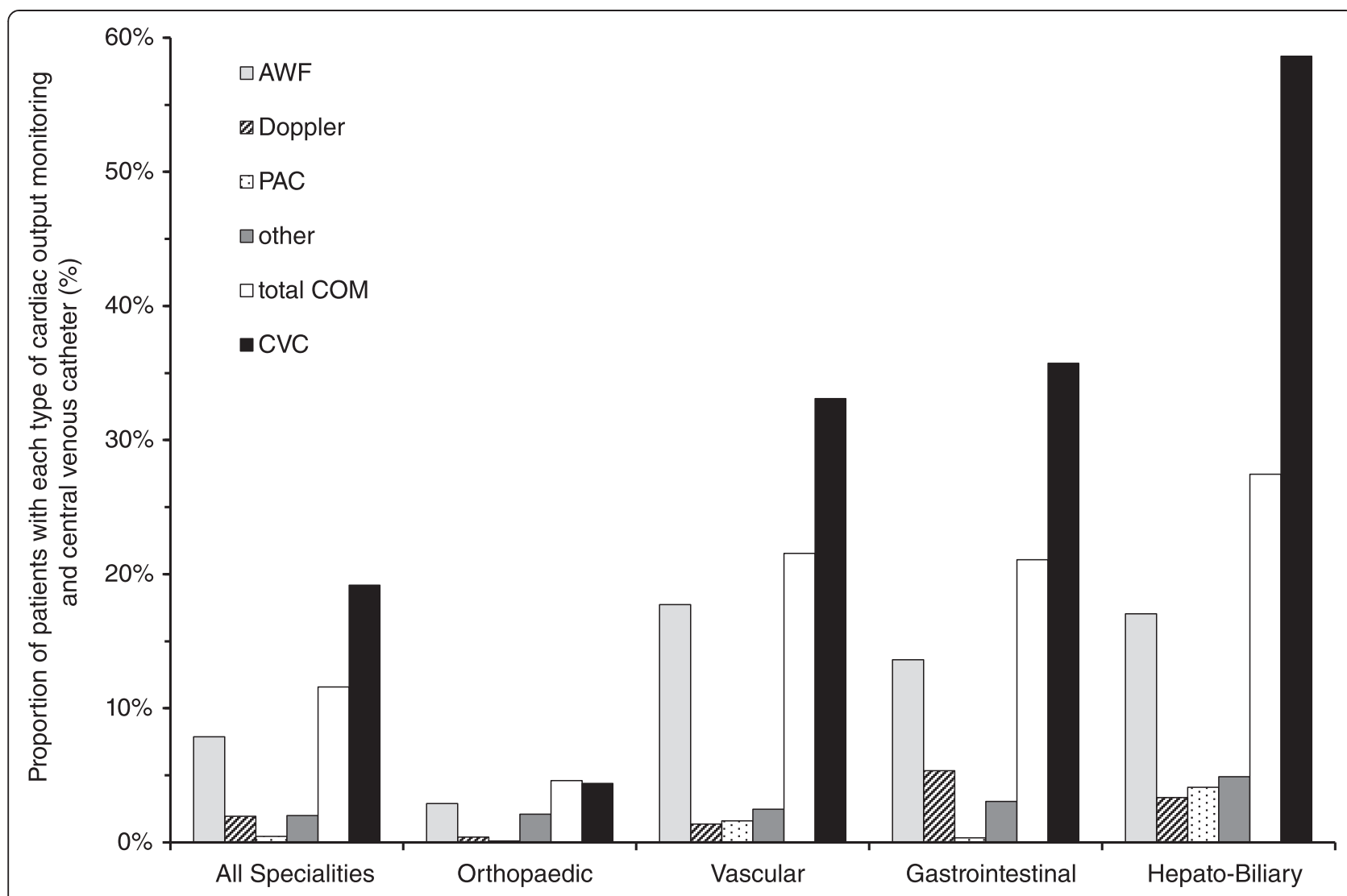

Fig. 2 Use of cardiac output monitoring and central venous catheter overall and per surgical speciality. Data displayed as percentage overall/per surgical speciality. AWF arterial waveform analysis, Doppler Doppler ultrasound, PAC pulmonary artery catheter, COM cardiac output monitoring, CVC central venous catheter

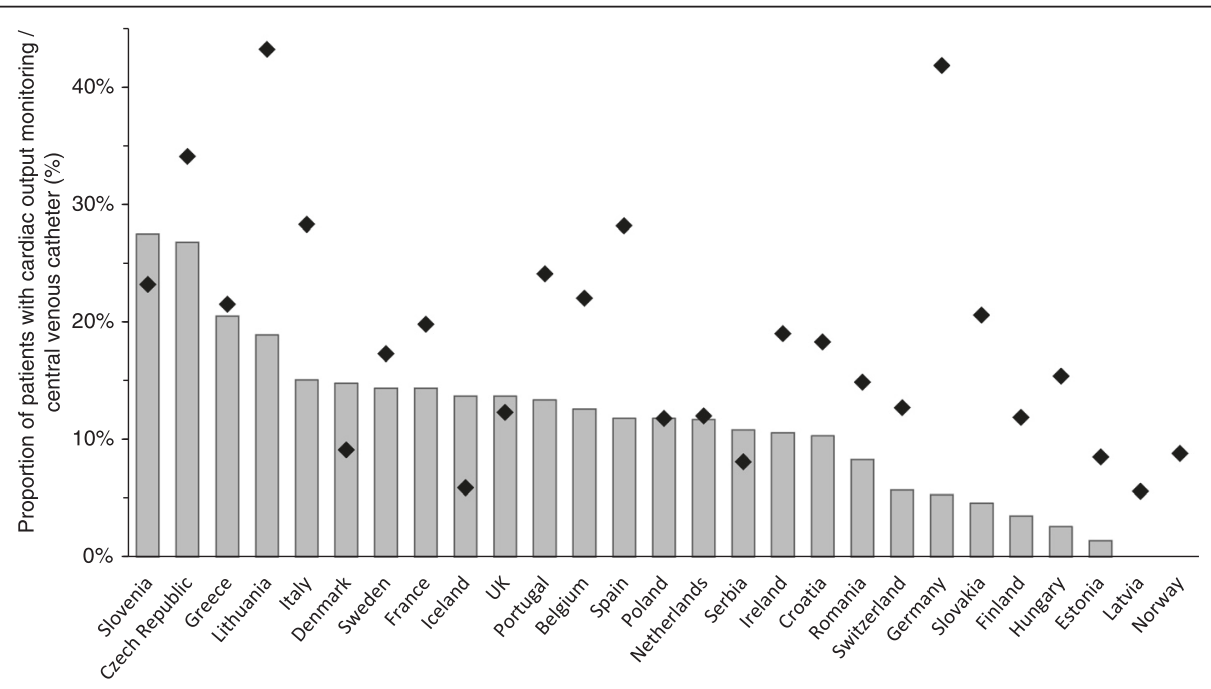

Fig. 3 Variation in use of cardiac output monitoring and central venous catheter in European nations. Data is presented in \% of patients with any type of cardiac output monitoring (bar) and central venous catheter (black diamond) per nation. Only nations with more than ten recruited patients are included into this graph. UK United Kingdom 
including availability of equipment, experience or education and national factors like reimbursement and guidelines [6, 21-23]. Involvement of clinicians in the development of, and therefore familiarity with, a certain device may also provide another explanation, which is why, for example, the use of PiCCO (PULSION Medical Systems, Germany) or LiDCO (LiDCO Ltd., United Kingdom) seems to be focused on their country of origin [6]. In addition, involvement of national opinion leaders in clinical trials improving the evidence base for certain devices might have contributed to the more extensive use of oesophageal Doppler monitoring in the United Kingdom compared to other nations [24-26]. Scepticism regarding possible benefits and harms is seen as another important contributor [6]. This in view of studies which suggested that fluid restriction might be equally beneficial as fluid optimization [19, 27, 28].

To our knowledge, this is the largest available patient dataset describing the use of haemodynamic monitoring across international boundaries. However, the study only provides evidence of activity in one 7-day period in participating centres and so may not provide an accurate reflection of activity over a longer time frame. The data were collected 4 years ago, and contemporary practice may have evolved since the original study. The small number of patients returned by a small number of hospitals in smaller countries may also have resulted in bias and a poor representation of patterns of monitoring use within those countries. In addition, the small patient numbers in some sub-categories limit the robustness of any analysis of variation between hospitals or potential clustering within hospitals, especially as some centres may specialise in particular types of surgery with consequent effects on care pathways and case mix. The study was not specifically designed for collection of data describing the use of haemodynamic monitoring and did not provide precise detail on the specific product used in each patient. No data were collected to describe how haemodynamic data were used, the target values for specific variables or which interventions were used to attain these targets. In addition, there is no data describing the indication for CVC placement. Due to the heterogeneity of cases and the limited sample size, we were not able to further analyse effects of haemodynamic monitoring on clinical outcomes.

\section{Conclusions}

One in ten patients undergoing major surgery in Europe is exposed to cardiac output monitoring whilst one in five receives a central venous catheter. The use of both technologies varies widely across nations. Further research is needed to confirm the clinical value of cardiac output monitoring and how this technology might help to further reduce postoperative morbidity and mortality.

\section{Additional file}

Additional file 1: Supplemental digital content. Patient flow diagram (supplementary Figure 1), variation in the use of different types of cardiac output monitoring in European nations (supplementary Figure 2) and types of haemodynamic monitoring used in European nations (supplementary Table). (PDF 333 kb)

\section{Abbreviations}

ASA: American Society of Anesthesiologists; AWF: arterial waveform analysis; COM: cardiac output monitoring; COPD: chronic obstructive pulmonary disease; CVC: central venous catheter; EuSOS: European Surgical Outcomes Study; ICU: intensive care unit; NICE: National Institute for Health and Care Excellence; NIDDM: non-insulin-dependent diabetes mellitus; PAC: pulmonary artery catheter; UK: United Kingdom.

\section{Competing interests}

ZM has received consulting fees and payment for lectures from PULSION Medical Systems, Biotest, ThermoFisher and CytoSorbent. $\mathrm{CH}$ has received grants and payment for lectures from PULSION Medical Systems and Edwards LifeSciences. MS has received consultancy fees from Masimo Inc. and Ratiopharm and grants from PULSION Medical Systems, Edwards LifeSciences and The Medicines Company, respectively, as payment for lectures from Edwards LifeSciences, PULSION Medical Systems, Fresenius Medical and Clearflow. AR has received payments for lectures from LiDCo Ltd., Masimo Inc. and Edwards LifeSciencies. RP has received equipment loans from LiDCO Ltd. and a research grant from Nestle Health Sciences. RP has performed consultancy work and/or given invited lectures for Nestle Health Sciences, Edwards LifeSciences and Massimo Inc. All other authors declare they have no conflicts of interest.

\section{Authors' contributions}

$T A, C B, C A, R M, Z M, V N, C H, M S, A R$ and RP were responsible for the study analysis and/or data interpretation. The manuscript was drafted by TA, CB and RP and revised following critical review by all authors. The authors received no other assistance in drafting this manuscript. All authors read and approved the final version of the manuscript.

\section{Acknowledgements}

The study was funded by the European Society of Intensive Care Medicine and the European Society of Anaesthesiology.

\section{Author details}

${ }^{1}$ Barts and The London School of Medicine and Dentistry, Queen Mary University of London, London, UK. ${ }^{2}$ Hospital Universitario Rio Hortega, Valladolid, Spain. ${ }^{3}$ Unidade de Cuidados Intensivos Neurocríticos, Centro Hospitalar de Lisboa Central, Lisbon, Portugal. ${ }^{4}$ University of Szeged, Szeged, Hungary. ${ }^{5}$ Ljubljana University Medical Centre, Ljubljana, Slovenia. ${ }^{6}$ Triemli City Hospital of Zurich, Zurich, Switzerland. ${ }^{7}$ Charité University, Berlin, Germany. ${ }^{8}$ St. George's University of London, London, UK. ${ }^{9}$ Adult Critical Care Unit, Royal London Hospital, London E1 1BB, UK.

Received: 4 July 2015 Accepted: 16 September 2015

Published online: 23 September 2015

\section{References}

1. Weiser TG, Haynes AB, Molina G, Lipsitz SR, Esquivel MM, Uribe-Leitz T, et al. Estimate of the global volume of surgery in 2012: an assessment supporting improved health outcomes. Lancet. 2015;385:S11.

2. Pearse RM, Moreno RP, Bauer P, Pelosi P, Metnitz P, Spies C, et al. Mortality after surgery in Europe: a 7 day cohort study. Lancet. 2012;380:1059-65.

3. Pearse RM, Harrison DA, James P, Watson D, Hinds C, Rhodes A, et al. Identification and characterisation of the high-risk surgical population in the United Kingdom. Crit Care. 2006;10:R81.

4. Pearse RM, Harrison DA, MacDonald N, Gillies MA, Blunt M, Ackland G, et al. Effect of a perioperative, cardiac output-guided hemodynamic therapy algorithm on outcomes following major gastrointestinal surgery: a randomized clinical trial and systematic review. JAMA. 2014;311:2181-90 
5. Pearse RM, Ackland GL. Perioperative fluid therapy. BMJ. 2012;344:e2865.

6. Cannesson M, Pestel G, Ricks C, Hoeft A, Perel A. Hemodynamic monitoring and management in patients undergoing high risk surgery: a survey among North American and European anesthesiologists. Crit Care. 2011;15:R197.

7. Srinivasa S, Kahokehr A, Soop M, Taylor M, Hill AG. Goal-directed fluid therapy- a survey of anaesthetists in the UK, USA, Australia and New Zealand. BMC Anesthesiol. 2013;13:5.

8. Kim S, Kim M, Lee J, Cho S, Chae W, Cannesson M. Current practice in hemodynamic monitoring and management in high-risk surgery patients: a national survey of Korean anesthesiologists. Korean J Anesthesiol. 2013;65:19-32.

9. Chen G, Zuo Y, Yang L, Chung E, Cannesson M. Hemodynamic monitoring and management of patients undergoing high-risk surgery: a survey among Chinese anesthesiologists. J Biomed Res. 2014;28:376-82.

10. Cecconi M, Corredor C, Arulkumaran N, Abuella G, Ball J, Grounds RM, et al. Clinical review: goal-directed therapy-what is the evidence in surgical patients? The effect on different risk groups. Crit Care. 2013;17:209.

11. Downs EA, Isbell JM. Impact of hemodynamic monitoring on clinical outcomes. Best Pract Res Clin Anaesthesiol. 2014;28:463-76.

12. Srinivasa S, Taylor MHG, Sammour T, Kahokehr AA, Hill AG. Oesophageal Doppler-guided fluid administration in colorectal surgery: critical appraisal of published clinical trials. Acta Anaesthesiol Scand. 2011;55:4-13.

13. Benes J, Giglio M, Brienza N, Michard F. The effects of goal-directed fluid therapy based on dynamic parameters on post-surgical outcome: a meta-analysis of randomized controlled trials. Crit Care. 2014;18:584.

14. Grocott MPW, Dushianthan A, Hamilton MA, Mythen MG, Harrison D, Rowan K. Perioperative increase in global blood flow to explicit defined goals and outcomes following surgery. Cochrane Database Syst Rev. 2012;11:CD004082.

15. Srinivasa S, Lemanu DP, Singh PP, Taylor MHG, Hill AG. Systematic review and meta-analysis of oesophageal Doppler-guided fluid management in colorectal surgery. Br J Surg. 2013;100:1701-8.

16. Chappell D, Jacob M, Hofmann-Kiefer K, Conzen P, Rehm M. A rational approach to perioperative fluid management. Anesthesiology. 2008;109:723-40.

17. Drummond GB, Soni N, Palazzo MGA. Prediction and theory in cardiac output estimates. Anaesthesia. 2010;65:751-2. author reply 752-3.

18. Soni N. Swan song for the Swan-Ganz catheter? BMJ. 1996;313:763-4.

19. Brandstrup $B$, Tønnesen $H$, Beier-Holgersen $R$, Hjortsø $E$, Ørding $H$, Lindorff-Larsen $\mathrm{K}$, et al. Effects of intravenous fluid restriction on postoperative complications: comparison of two perioperative fluid regimens: a randomized assessor-blinded multicenter trial. Ann Surg. 2003;238:641-8

20. Challand C, Struthers R, Sneyd JR, Erasmus PD, Mellor N, Hosie KB, et al. Randomized controlled trial of intraoperative goal-directed fluid therapy in aerobically fit and unfit patients having major colorectal surgery. BJA. 2012;108:53-62.

21. National Institute for Health and Clinical Excellence. CardioQ-ODM Oesophageal Doppler Monitor. March 2011. http://www.nice.org.uk/ guidance/MTG3. Accessed: 14 January 2015.

22. Agency for Healthcare Research and Quality. Esophageal Doppler ultrasound-based cardiac output monitoring for real-time therapeutic management of hospitalized patients: a review. January 2007. http://www.cms.gov/determinationprocess/downloads/id45TA.pdf. Accessed: 14 January 2015.

23. Lassen K, Soop M, Nygren J, Cox PBW, Hendry PO, Spies C, et al. Consensus review of optimal perioperative care in colorectal surgery: Enhanced Recovery After Surgery (ERAS) Group recommendations. Arch Surg. 2009;144:961-9.

24. Sinclair S, James $S$, Singer $M$. Intraoperative intravascular volume optimisation and length of hospital stay after repair of proximal femoral fracture: randomised controlled trial. BMJ. 1997;315:909-12.

25. Wakeling HG, McFall MR, Jenkins CS, Woods WGA, Miles WFA, Barclay GR, et al. Intraoperative oesophageal Doppler guided fluid management shortens postoperative hospital stay after major bowel surgery. BJA. 2005;95:634-42.

26. Noblett SE, Snowden CP, Shenton BK, Horgan AF. Randomized clinical trial assessing the effect of Doppler-optimized fluid management on outcome after elective colorectal resection. Br J Surg. 2006;93:1069-76.
27. Brandstrup B, Svendsen PE, Rasmussen M, Belhage B, Rodt SÅ, Hansen B, et al. Which goal for fluid therapy during colorectal surgery is followed by the best outcome: near-maximal stroke volume or zero fluid balance? BJA. 2012;109:191-9.

28. Srinivasa S, Taylor MHG, Singh PP, Yu T, Soop M, Hill AG. Randomized clinical trial of goal-directed fluid therapy within an enhanced recovery protocol for elective colectomy. Br J Surg. 2013;100:66-74.

\section{Submit your next manuscript to BioMed Central and take full advantage of:}

- Convenient online submission

- Thorough peer review

- No space constraints or color figure charges

- Immediate publication on acceptance

- Inclusion in PubMed, CAS, Scopus and Google Scholar

- Research which is freely available for redistribution 